\title{
LINEAR PARAMETER VARYING CONTROL SYNTHESIS FOR ACTUATOR FAILURE, BASED ON ESTIMATED PARAMETER *
}

\author{
Jong-Yeob Shin, ${ }^{\dagger}$ N. Eva Wu, ${ }^{\ddagger}$ and Christine Belcastro ${ }^{\S}$
}

\begin{abstract}
The design of a linear parameter varying (LPV) controller for an aircraft at actuator failure cases is presented. The controller synthesis for actuator failure cases is formulated into linear matrix inequality (LMI) optimizations based on an estimated failure parameter with pre-defined estimation error bounds. The inherent conservatism of an LPV control synthesis methodology is reduced using a scaling factor on the uncertainty block which represents estimated parameter uncertainties. The fault parameter is estimated using the two-stage Kalman filter. The simulation results of the designed LPV controller for a HiMAT (Highly Maneuverable Aircraft Technology) vehicle with the on-line estimator show that the desired performance and robustness objectives are achieved for actuator failure cases.
\end{abstract}

\section{Introduction}

A fault tolerant control (FTC) system is one of the important research areas for a single aircraft control upset prevention. A FTC system requires to detect sensor/actuator faults and to reconfigure control laws according to faults. Also, fault detection and isolation (FDI) mechanism in a FTC system should properly integrate with a fault tolerant controller which can be designed as a reconfigurable controller. A fault tolerance controller may degrade

\footnotetext{
* Copyright (C) 2002 by J-Y. Shin, N. E. Wu, and C. Belcastro. Published by the American Insitute of Aeronautics and Astronautics, Inc. with permission.

† Staff Scientist, ICASE, NASA Langley Research Center, Hampton, VA 23681. AlAA Member

$\ddagger$ Professor, Department of Electrical Engineering, Binghamton State University of New York, Binghamton, New York, 13902.

$\S$ Researcher, Guidance Control Brench, NASA Langley Research Center, Hampton, VA 23681.
}

performance but should prevent fetal failure of a aviation system for fault accommodation.

Numerous research in the field of a FTC system has been done. ${ }^{1-3}$ In Ref. $[2,3]$, a fault tolerant controller is designed based on a priori information on possible faults which can be modeled as a function of parameters. When possible faults are modeled as a linear parameter varying (LPV) system, LPV control synthesis is naturally fit into designing a fault tolerant controller. An LPV control synthesis is formulated into a linear matrix inequality (LMI) optimization based on an LPV system whose state-space matrices are functions of a scheduling parameter vector. A scheduling parameter vector should be measurable at current time and its rate is bounded..$^{4,5}$ The LPV controller robustly stabilize the close-loop system and achieve the desired performance level over the entire parameter space. ${ }^{4,5}$ The LPV synthesis methodology has been successfully applied to synthesis controllers for the pitch-axis missile autopilots, ${ }^{6,7}$ F-14 aircraft lateral-directional axis during powered approach, ${ }^{8}$ turbofan engines ${ }^{9}$ and F-16 aircraft. ${ }^{10}$

In this paper, a fault tolerant controller is designed using an LPV control synthesis methodology. However, the fault parameter can not be directly measured via sensors at current time, since the fault parameter is not a physical quantity. Using a FDI mechanism, the fault parameters can be estimated with a bounded error. ${ }^{11}$ Estimation errors always exist in a real control system due to unmodeled dynamics, disturbances, and measurement sensor errors. Thus, parameter estimation errors should be considered in a fault-tolerant control design process.

In this paper, fault estimation errors and modeling uncertainties are represented by an uncertainty block in the construct of a LPV controller. The structure of an uncertainty block is included in a robust LPV control synthesis methodology. The robust LPV control synthesis is formulated into two LMI optimization problems which can be solved by 
an iteration method. ${ }^{4}$

In this paper, actuator failures are modeled as an LPV system as functions of actuator effectiveness parameters. ${ }^{11}$ These parameters are estimated as biases using an augmented Kalman filter. A set of covariance-dependent forgetting factors is introduced into the filtering algorithm. As a result, the change in the actuator effectiveness is accentuated to help achieve a more accurate estimate more rapidly. The $H_{\infty}$ bounds on parameter estimation errors are assessed through simulations, which are then used as bounds of real parameter uncertainty in the construction of a robust LPV control law. Actuator faults can be parameterized as estimated fault effectiveness parameters. Thus, it is possible to formulate a fault tolerance control design problem as an LPV control synthesis problem based on estimated faults parameters.

This paper contains the following sections. In Section 2, an robust LPV synthesis methodology used in this paper is summarized. In Section 3, fault parameter estimation methods are presented. In Section 4, an LPV controller for a HiMAT vehicle is designed by using an robust LPV synthesis control methodology. In Section 5, the simulation results of the closed-loop system with an on-line estimator are presented and this paper concludes with a brief summary in Section 6 .

\section{LPV Synthesis}

\subsection{Problem Statements}

In this section, a control synthesis problem is defined, based on an estimated parameter vector $\bar{\rho} \epsilon$ $\mathcal{R}^{n}$, such as actuator effectiveness. ${ }^{11}$ Actuator effectiveness parameters represent actuator failure cases (actuator damage) which can be estimated using the estimation methods presented in Section 3.

An LPV system can be represented as functions of an estimated scheduling parameter vector $\bar{\rho}$ with an uncertainty block $\Delta$ which captures parameter estimation errors and unmodeled dynamics. An LPV system can be written as:

$$
\begin{gathered}
{\left[\begin{array}{c}
\dot{x} \\
e_{\Delta} \\
e_{p} \\
y
\end{array}\right]=\left[\begin{array}{cccc}
A(\bar{\rho}) & B_{\Delta}(\bar{\rho}) & B_{p}(\bar{\rho}) & B_{u}(\bar{\rho}) \\
C_{e}(\bar{\rho}) & D_{\Delta \Delta}(\bar{\rho}) & D_{\Delta p}(\bar{\rho}) & D_{\Delta u}(\bar{\rho}) \\
C_{p}(\bar{\rho}) & D_{p \Delta}(\bar{\rho}) & D_{p p}(\bar{\rho}) & D_{p u}(\bar{\rho}) \\
C_{y}(\bar{\rho}) & D_{y \Delta}(\bar{\rho}) & D_{y p}(\bar{\rho}) & 0
\end{array}\right]\left[\begin{array}{c}
x \\
d_{\Delta} \\
d_{p} \\
u
\end{array}\right]} \\
d_{\Delta}=\Delta e_{\Delta}
\end{gathered}
$$

where $x \in \mathcal{R}^{n_{x}}, e_{\Delta} \in \mathcal{R}^{n_{\Delta}}, e_{p} \in \mathcal{R}^{n_{e_{p}}}, y \in \mathcal{R}^{n_{y}}$, $d_{\Delta} \in \mathcal{R}^{n_{\Delta}}, d_{p} \in \mathcal{R}^{n_{d_{p}}}$, and $u \in \mathcal{R}^{n_{u}}$. All of the state-space matrices are of appropriate dimensions.

An uncertainty block set $\Delta$ is defined as:

$\Delta=\left\{\begin{array}{l}\Delta=\operatorname{diag}\left(\delta_{1} I_{1}, \cdots, \delta_{n} I_{n}, \Delta_{n+1}, \cdots, \Delta_{n+m}\right) \\ : \delta_{i} \in R, \Delta_{i} \in R^{l \times l}, \bar{\sigma}(\Delta) \leq \beta\end{array}\right\}$

where $\beta$ is normalized to 1 without loss of generality. There exists a scaling factor set $S$ such that

$$
\boldsymbol{S}=\left\{S: S>0, \quad S \Delta=\Delta S, \quad S \in \mathcal{R}^{n_{\Delta} \times n_{\Delta}}\right\} .
$$

The input/output scaling matrices $L^{-1 / 2}$ and $J^{1 / 2}$ are defined as

$$
\begin{aligned}
L^{-1 / 2} & =\left[\begin{array}{cc}
S^{-1 / 2} & 0 \\
0 & I_{n_{d_{p}}}
\end{array}\right] \in \mathcal{R}^{\left(n_{\Delta}+n_{d_{p}}\right) \times\left(n_{\Delta}+n_{d_{p}}\right)}, \\
J^{1 / 2} & =\left[\begin{array}{cc}
S^{1 / 2} & 0 \\
0 & I_{n_{e_{p}}}
\end{array}\right] \in \mathcal{R}^{\left(n_{\Delta}+n_{e_{p}}\right) \times\left(n_{\Delta}+n_{e_{p}}\right)} .
\end{aligned}
$$

The induced $\mathcal{L}_{2}$-norm of a parameter dependent stable LPV system is defined as

$$
\|G\|_{2 \leftarrow 2} \equiv \sup _{\forall \bar{\rho}, d \in \mathcal{L}_{2}, d \neq 0} \frac{\|e\|_{2}}{\|d\|_{2}}
$$

for zero initial conditions $x(0)=0$.

The control synthesis problem is to design an LPV controller $K(\bar{\rho})$ and scaling factor $S$ in order to minimize the induced $\mathcal{L}_{2}$ norm of the closed-loop system. The robust LPV control synthesis can be formulated into an optimization problem:

$$
\min _{K(\bar{\rho}), S \in S}\left\|J^{1 / 2}(S) F_{l}(P(\bar{\rho}), K(\bar{\rho})) L^{-1 / 2}(S)\right\|_{2 \leftarrow 2}
$$

where $F_{l}(P(\bar{\rho}), K(\bar{\rho}))$ means a lower linear fraction transformation (LFT). The optimization problem of equation $(2)$ is not convex in $K(\bar{\rho})$ and $S$. When a estimated scheduling parameter $\bar{\rho}$ is fixed, the problem is same as a D-K iteration problem. ${ }^{12}$ In this paper, the robust LPV control synthesis is formulated into two LMI optimization problems which can be solved using an iteration procedure.

\subsection{Control Synthesis Methodology}

In this section, an iteration procedure of solving the problem is presented. There is an LPV control synthesis methodology in Ref. $\left[{ }^{4}\right]$ with a scaling factor. In Ref. $\left[{ }^{4}\right]$, an LMI optimization can be formulated with unknown matrices $X>0, Y>0$, and scaling factor matrices $J^{1 / 2}$ and $L^{-1 / 2}$. However, an 
LMI optimization problem in Ref. $\left.{ }^{4}\right]$ has an equality constraint. In this paper, to avoid an equality constraint, the augment LPV system with the scaling matrices $J^{1 / 2}$ and $L^{-1 / 2}$ is used to design an LPV controller.

Suppose a scaling factor $S$ is given. Hereafter, $\bar{\rho}$ dependence in matrices is omitted for space limitation. The augmented LPV open-loop system with the scaling matrices $J^{1 / 2}$ and $L^{-1 / 2}$ can be written as:

$$
\left[\begin{array}{l}
\dot{x} \\
e \\
y
\end{array}\right]=\left[\begin{array}{ccc}
A & B_{1} L^{-1 / 2} & B_{2} \\
J^{1 / 2} C_{1} & J^{1 / 2} D_{11} L^{-1 / 2} & J^{1 / 2} D_{12} \\
C_{2} & D_{21} L^{-1 / 2} & 0
\end{array}\right]\left[\begin{array}{l}
x \\
d \\
u
\end{array}\right]
$$

where $e=\left[\begin{array}{ll}e_{\Delta}^{T} & e_{p}^{T}\end{array}\right]^{T}$ and $d=\left[\begin{array}{ll}d_{\Delta}^{T} & d_{p}^{T}\end{array}\right]^{T}$. With assumption that $\bar{D}_{12}$ and $\bar{D}_{21}$ are full column and row rank for all $\bar{\rho}$, respectively, an LPV control synthesis methodology in Ref. $\left.{ }^{5,13}\right]$ can be used in this paper.

For the sake of completeness, a brief summary of the LPV control synthesis methodology in Ref.[5] is presented in this section. There exists an LPV controller $K(\bar{\rho})$ which leads the induced- $\mathcal{L}_{2}$ norm of the closed-loop system is less than constant $\gamma$. The LPV controller $K(\bar{\rho})$ can be constructed from the solution matrices, $X(\bar{\rho}) \in \mathcal{R}^{n \times n}$ and $Y(\bar{\rho}) \in \mathcal{R}^{n \times n}$ which are calculated by solving the following LMI optimization.

$$
\min _{x(\tilde{\rho}), Y(\bar{\rho})} \gamma
$$

subject to the LMI constraints defined in Ref. $\left[{ }^{5}\right]$. The realization of an LPV controller from the solution matrices $X$ and $Y$ are taken from $\left.\operatorname{Ref} .{ }^{5}\right]$.

The benefit of the LPV synthesis methodology is that there is no limitation of an affine functional form of LPV system state-space matrices. Since the LMI constraints are evaluated at grid points over all scheduling parameter spaces, an LPV system should be just a continuous and differentiable function of a scheduling parameter. The disadvantage of the LPV control synthesis methodology is that robust stability over all parameter spaces is not guaranteed unless choosing appropriate number of grid points.

Suppose there exists an designed LPV controller $K(\bar{\rho})$ which stabilizes the augmented LPV system. The closed-loop LPV system with a given controller is written as:

$$
\left[\begin{array}{c}
\dot{x}_{c l} \\
e_{\Delta} \\
e_{p}
\end{array}\right]=\left[\begin{array}{ccc}
A_{c l} & \tilde{B}_{1 c l} & B_{2_{c l}} \\
\tilde{C}_{1_{c l}} & \tilde{D}_{11_{c l}} & \bar{D}_{21_{c l}} \\
C_{2_{c l}} & \tilde{D}_{21_{c l}} & D_{22_{c l}}
\end{array}\right]\left[\begin{array}{c}
x_{c l} \\
d_{\Delta} \\
d_{p}
\end{array}\right], d_{\Delta}=\Delta e_{\Delta}
$$

where $x_{c l}=\left[\begin{array}{ll}x^{T} & x_{k}^{T}\end{array}\right]^{T}, \quad \tilde{B}_{1_{c l}}=B_{1_{c l}} S^{-1 / 2}$, $\tilde{C}_{1_{c l}}=S^{1 / 2} C_{1_{c l},}, \tilde{D}_{11_{c l}}=S^{1 / 2} D_{11_{c l}} S^{-1 / 2}, \tilde{D}_{21_{c l}}=$ $S^{1 / 2} D_{21_{c l}}$, and $D_{21_{c l}}=D_{21_{c l}} S^{-1 / 2}$.
Applying the Kalman-Yakubovich-Popov (KYP) Lemma, ${ }^{11}$ the LMI optimization is formulated to find a scaling factor $S$. There exists an scaling factor $S \in S$ which leads the induced- $\mathcal{L}_{2}$ norm of the closed-loop system is less than $\gamma_{s}$. The scaling factor $S$ can be determined solving the following LMI optimization.

$$
\min _{P>0, S \in S} \gamma_{s}
$$

subject to

$$
\begin{aligned}
& {\left[\begin{array}{ccc}
A^{T} P+P A+\dot{P} & P B_{1_{c l}} & P B_{2_{c l}} \\
B_{1 c l}^{T} P & -S & 0 \\
B_{2 c l}^{T} P & 0 & -I
\end{array}\right]} \\
& +\left[\begin{array}{cc}
C_{1_{c l}}^{T} & C_{2_{c l}}^{T} \\
D_{11_{c l}}^{T} & D_{21_{c l}}^{T} \\
D_{12_{c l}}^{T} & D_{22_{c l}}^{T}
\end{array}\right]\left[\begin{array}{ccc}
S C_{1_{c l}} & S D_{11_{c l}} & S D_{12_{c l}} \\
\gamma^{-1} C_{2_{c l}} & \gamma^{-1} D_{21_{c l}}^{T} & \gamma^{-1} D_{22_{c l}}^{T}
\end{array}\right]<0
\end{aligned}
$$

The iteration procedure to solve the problem in equation (2) is follows:

1. Design an LPV controller $K(\bar{\rho})$ for a system from the LMI optimization in equation (4) with fixed $S$. At the first iteration, $S$ is assumed as I.

2. Solve the LMI optimization problem in equation (5) over $P(\bar{\rho})$ and $S$ based on the closedloop system with the designed LPV controller $K(\bar{\rho})$.

3. Generate an augmented LPV system with the scaling factor $S$

$$
G_{i+1}(\bar{\rho})=\left[\begin{array}{cc}
S^{1 / 2} & 0 \\
0 & I
\end{array}\right] G_{i}(\bar{\rho})\left[\begin{array}{cc}
S^{-1 / 2} & 0 \\
0 & I
\end{array}\right]
$$

where $G_{i}(\vec{\rho})$ is an LPV model at the $i^{\text {th }}$ iteration.

4. Iterate over step 1 to 3 until convergence or terminate iteration based on satisfaction with a designed LPV controller.

The iteration method can not guarantee finding global solutions of $K$ and $S$ since the problem in equation (2) is not convex in $K$ and $S$. Also, there is no guarantee of convergence in the iteration process. When a designed controller is fixed to calculate the scaling factor $S$, the matrix $P(\bar{\rho})$ can be calculated from the solution matrices $X$ and $Y$ and fixed in the LMI optimization. ${ }^{5}$ Note that the matrix $P(\bar{\rho})$ is also set as an unknown matrix in the LMI optimization in equation (5) in this paper, to relax the constraints of fixing the LPV controller $K(\bar{\rho})$.

3 


\section{Parameter Estimation}

This section briefly describes the formulation of a real parameter estimation problem, which, when specialized to the actuator effectiveness estimation, transforms a fault (loss of actuator effectiveness) tolerant control problem to a robust LPV control problem. The development of this section follows that in Ref. $\left[{ }^{11}\right]$.

The estimator is based on a linear discrete design model of the form:

$$
\begin{aligned}
x_{k+1}= & A_{k}^{d} x_{k}+\left[\begin{array}{lll}
b_{1}^{d} \gamma_{k}^{1} & \cdots & b_{m}^{d} \gamma_{k}^{m}
\end{array}\right]\left[\begin{array}{c}
u_{k}^{1} \\
u_{k}^{2} \\
\vdots \\
u_{k}^{m}
\end{array}\right] \\
& +B_{k}^{d} u_{k}+w_{k}^{x} \\
= & A_{k}^{d} x_{k}+E_{k}^{d} \gamma_{k}+B_{k}^{d} u_{k}+w_{k}^{x} \\
\gamma_{k+1}= & \gamma_{k}+w_{k}^{\gamma} \\
y_{k}= & C_{k}^{d} x_{k}+v_{k},
\end{aligned}
$$

where $x_{k} \in R^{n_{x}}, \gamma_{k} \in R^{n_{u}}, u_{k} \in R^{n_{u}}$ and $y_{k} \in R^{n_{y}}$ are the state, bias, input, and output variables, respectively. The discrete model can be obtained from a continuous model via, for example, the Euler's rule with a sampling period $T_{s}$, which preserves the functional dependence of the " $B$ " matrix on $\gamma$. The bias vector $\gamma$ with component $-1 \leq \gamma_{k}^{i} \leq 0$ relates to a actuator failure parameter. It is obvious that $E_{k}^{d}=B_{k}^{d} \times \operatorname{diag}\left\{u_{k}^{1}, \cdots, u_{k}^{n_{u}}\right\} \cdot w_{k}^{x}, w_{k}^{\gamma}$ and $v_{k}$ denote the white noise sequences of uncorrelated Gaussian random vectors with zero means and covariance matrices $Q_{k}^{x}, Q_{k}^{\gamma}$ and $R_{k}$, respectively.

The minimum variance solution is obtained by a direct application of the two-stage Kalman filter algorithm of Keller and Darouach, ${ }^{14}$ with constant coefficient matrices in Ref. $\left[{ }^{14}\right]$ replaced by time-varying matrices. The filter is decoupled into four sets of equations. They given as follows.

-Optimal bias estimator

$$
\begin{aligned}
\hat{\gamma}_{k+1 \mid k}= & \hat{\gamma}_{k \mid k}, \\
P_{k+1 \mid k}^{\gamma}= & P_{k \mid k}^{\gamma}+Q_{k}^{\gamma} \\
\hat{\gamma}_{k+1 \mid k+1}= & \hat{\gamma}_{k+1 \mid k}+K_{k+1}^{\gamma}\left(\tilde{r}_{k+1}-H_{k+1 \mid k} \hat{\gamma}_{k \mid k}\right) \\
K_{k+1}^{\gamma}= & P_{k+1 \mid k}^{\gamma} H_{k+1 \mid k}^{T} \\
& \left(H_{k+1 \mid k} P_{k+1 \mid k}^{\gamma} H_{k+1 \mid k}^{T}+\tilde{S}_{k+1}\right)^{-1} \\
P_{k+1 \mid k+1}^{\gamma}= & \left(I-K_{k+1}^{\gamma} H_{k+1 \mid k}\right) P_{k+1 \mid k}^{\gamma} .
\end{aligned}
$$

-Bias-free state estimator

$$
\begin{aligned}
\tilde{x}_{k+1 \mid k}= & A_{k}^{d} \tilde{x}_{k \mid k}+B_{k}^{d} u_{k}+W_{k} \hat{\gamma}_{k \mid k}-V_{k+1 \mid k} \hat{\gamma}_{k \mid k}, \\
\tilde{P}_{k+1 \mid k}^{x}= & A_{k}^{d} \tilde{P}_{k \mid k}^{x}\left(A_{k}^{d}\right)^{T}+Q_{k}^{x}+W_{k} P_{k \mid k}^{\gamma} W_{k}^{T} \\
& -V_{k+1 \mid k} P_{k+1 \mid k}^{\gamma} V_{k+1 \mid k}^{T}, \\
\tilde{x}_{k+1 \mid k+1}= & \tilde{x}_{k+1 \mid k}+\tilde{K}_{k+1}^{x}\left(y_{k+1}-C_{k+1}^{d} \tilde{x}_{k+1 \mid k}\right), \\
\tilde{K}_{k+1}^{x}= & \tilde{P}_{k+1 \mid k}^{x}\left(C_{k+1}^{d}\right)^{T}\left\{C_{k+1}^{d} \tilde{P}_{k+1 \mid k}^{x}\left(C_{k+1}^{d}\right)^{T}\right. \\
& \left.+R_{k+1}\right\}^{-1}, \\
\tilde{P}_{k+1 \mid k+1}^{x}= & \left(I-\tilde{K}_{k+1}^{x} C_{k+1}^{d}\right) \tilde{P}_{k+1 \mid k}^{x},
\end{aligned}
$$

where the filter residual and its covariance are given as

$$
\begin{aligned}
& \tilde{r}_{k+1}=y_{k+1}-C_{k+1}^{d} \tilde{x}_{k+1 \mid k}, \\
& \tilde{S}_{k+1}=C_{k+1}^{d} \tilde{P}_{k+1 \mid k}^{x}\left(C_{k+1}^{d}\right)^{T}+R_{k+1} .
\end{aligned}
$$

-Coupling equations

$$
\begin{aligned}
W_{k} & =A_{k}^{d} V_{k \mid k}+E_{k}^{d}, \\
V_{k+1 \mid k} & =W_{k} P_{k \mid k}^{\gamma}\left(P_{k+1 \mid k}^{\gamma}\right)^{-1} \\
H_{k+1 \mid k} & =C_{k+1}^{d} V_{k+1 \mid k} \\
V_{k+1 \mid k+1} & =V_{k+1 \mid k}-\tilde{K}_{k+1}^{x} H_{k+1 \mid k} .
\end{aligned}
$$

-And finally the compensated state and error covariance estimates

$$
\begin{aligned}
& \hat{x}_{k+1 \mid k+1}=\tilde{x}_{k+1 \mid k+1}+V_{k+1 \mid k+1} \hat{\gamma}_{k+1 \mid k+1}, \\
& P_{k+1 \mid k+1}=\tilde{P}_{k+1 \mid k+1}^{x}+V_{k+1 \mid k+1} P_{k+1 \mid k+1}^{\gamma} V_{k+1 \mid k+1}^{T} .
\end{aligned}
$$

A further measure is taken to modify the above filtering algorithm so that the estimates become more responsive to abrupt changes in the control effectiveness factors.

A well known technique for estimating timevarying parameters is the use of forgetting factors. The basic idea is to enable a recursive algorithm to discount the past information so that the filter is more apt to recognize the changes in the system. Since the time update of the bias estimate governed by $\hat{\gamma}_{k+1 \mid k}=\hat{\gamma}_{k \mid k}$ is the dominant opposing force to acknowledge the abrupt changes in the biases, forgetting factors introduced into the time propagation equation $P_{k+1 \mid k}^{\gamma}=P_{k \mid k}^{\gamma}+Q_{k}^{\gamma}$ of the bias covariance is likely to function most effectively.

Assume that covariance $P_{k \mid k}^{\gamma}$ adequately describes the bias estimation error along both temporal and spacial directions under the normal system operation condition. Then this covariance provides a basis for the selection of forgetting factors. The bias estimates should be prevented from being impetuous, as well as from being indifferent to the 
changes shown in the measurements. A technique suggested in Ref. $\left.{ }^{15}\right]$ amounts to select forgetting factors that would force the adjusted covariance in $P_{k+1 \mid k}^{\gamma}=P_{k \mid k}^{\gamma}+Q_{k}^{\gamma}$ to stay within some prescribed bounds

$$
\sigma_{\min } I \leq P_{k+1 \mid k}^{\gamma} \leq \sigma_{\max } I
$$

where $\sigma_{\min }$ and $\sigma_{\max }$ are positive constants with $0<$ $\sigma_{\min }<\sigma_{\max }<\infty$, and $I$ is the identity matrix. Let the dyadic expansion of $P_{k \mid k}^{\gamma}$ be given by

$$
P_{k \mid k}^{\gamma}=\sum_{i=1}^{n_{u}} \alpha_{k \mid k}^{i} e_{k}^{i}\left(e_{k}^{i}\right)^{T},
$$

where $\alpha_{k \mid k}^{1}, \ldots, \alpha_{k \mid k}^{n_{u}}$ are the eigenvalues of $P_{k \mid k}^{\gamma}$ with $\alpha_{k \mid k}^{1} \geq \ldots \geq \alpha_{k \mid k}^{n_{u}}$, and $e_{k}^{1}, \ldots, e_{k}^{n_{u}}$ are the corresponding eigenvectors with $\left\|e_{k}^{1}\right\|=\ldots=\left\|e_{k}^{n_{u}}\right\|=1$. Equation (12) can then be expressed as

$$
P_{k+1 \mid k}^{\gamma}=\sum_{i=1}^{n_{u}} \frac{\alpha_{k \mid k}^{i}}{\lambda_{k}^{i}} e_{k}^{i}\left(e_{k}^{i}\right)^{T}+Q_{k}^{\gamma}, \quad 0<\lambda_{k}^{i} \leq 1 .
$$

Following the argument in Ref. $\left[{ }^{15}\right]$, the forgetting factor $\lambda_{k}^{i}$ can be chosen as a decreasing function of the amount of information received in the direction $e_{k}^{i}$. Since eigenvalue $\alpha_{k \mid k}^{i}$ of $P_{k \mid k}^{\gamma}$ is a measure of the uncertainty in the direction of $e_{k}^{i}$, a choice of forgetting factor $\lambda_{k}^{i}$ based on the above constraints can be

$\lambda_{k}^{i}= \begin{cases}\lambda_{o}, & \alpha_{k \mid k}^{i}>\alpha_{\max }, \\ \alpha_{k \mid k}^{i}\left[\alpha_{\min }+\frac{\alpha_{\max }-\alpha_{\min }}{\alpha_{\max }} \alpha_{k \mid k}^{i}\right]^{-1}, & \alpha_{k \mid k}^{i} \leq \alpha_{\max } .\end{cases}$

The estimation algorithm discussed in this section will be seen to have been applied successfully to a HiMAT model.

\section{Control of HiMAT Vehicle}

In this section, the robust LPV control synthesis methodology described in Section 2 is applied to control a HiMAT vehicle for actuator failure cases. Recall it is assumed that actuators are failed one at a time. Thus, the control reconfigurability of the HiMAT vehicle never goes to zero over the entire failure cases. ${ }^{16}$ The system variations due to actuator failures can be modeled as an LPV system, a function of an estimated scheduling parameter.

\subsection{LPV Model of HiMAT Vehicle}

The model of the HiMAT vehicle taken from the $\mu$ synthesis Toolbox ${ }^{12}$ has two inputs: elevons $\delta_{e}$ and canards $\delta_{c}$; two outputs: angle of attack $\alpha$ in radians and pitch angle $\theta$ in radians; and four states: velocity $V$ in $\mathrm{ft} / \mathrm{sec}$, angle of attack, pitch rate $q$ in $\mathrm{rad} / \mathrm{sec}$, and pitch angle. The open-loop model is

$$
\left[\begin{array}{l}
\dot{x} \\
y
\end{array}\right]=\left[\begin{array}{ll}
A & B \\
C & 0
\end{array}\right]\left[\begin{array}{l}
x \\
u
\end{array}\right], u=\left[\begin{array}{l}
\delta_{e} \\
\delta_{c}
\end{array}\right]
$$

where

$$
\begin{aligned}
A & =\left[\begin{array}{cccc}
-0.0226 & -36.6 & -18.9 & -32.1 \\
0 & -1.9 & 0.98 & 0 \\
0.012 & -11.7 & -2.63 & 0 \\
0 & 0 & 1 & 0
\end{array}\right], \\
B & =\left[\begin{array}{cc}
0 & 0 \\
-0.414 & 0 \\
-77.8 & 22.4 \\
0 & 0
\end{array}\right], C=\left[\begin{array}{llll}
0 & 1 & 0 & 0 \\
0 & 0 & 0 & 1
\end{array}\right] .
\end{aligned}
$$

A failure parameter vector $\tau=\left[\begin{array}{ll}\tau_{1} & \tau_{2}\end{array}\right]^{T}$ is determined by the two actuator effectiveness parameters $\tau_{1}$ and $\tau_{2}$ of elevon and canard actuators, respectively. Assume that the failure parameters linearly enter in the model. The state-space model of the HiMAT vehicle is written as

$$
\dot{x}=A x+B(\tau) u, \quad y=C x,
$$

where $A$ and $C$ are constant matrices and $B(\tau)=$ $\left[\begin{array}{ll}b_{1} \tau_{1} & b_{2} \tau_{2}\end{array}\right]$. The vectors $b_{1}$ and $b_{2}$ are the columns of $B$. The actuator failure parameters can be estimated using the estimation method described in Section 3. However, there is estimation error $\delta_{\tau}=$ $\left[\begin{array}{ll}\delta_{\tau_{1}} & \delta_{\tau_{2}}\end{array}\right]^{T}$. The actuator failure parameter vector $\tau$ is written as

$$
\tau=\bar{\tau}+\delta_{\tau},
$$

where $\bar{\tau}$ is an estimated value. The estimated actuator failure parameter can be written as:

$$
\begin{aligned}
& \bar{\tau}_{1}=1+\gamma^{1}, \\
& \bar{\tau}_{2}=1+\gamma^{2} .
\end{aligned}
$$

where $\gamma^{1}$ and $\gamma^{2}$ are bias parameters in equation (9). The estimation error bound is assumed as $\sqrt{\delta_{\tau}^{T} \delta_{\tau}} \leq$ 0.05 for each actuator failure case. The matrix $B(\tau)$ is rewritten as:

$$
B(\tau)=B\left[\begin{array}{cc}
\bar{\tau}_{1}+0.05 \delta_{1} & 0 \\
0 & \bar{\tau}_{2}+0.05 \delta_{2}
\end{array}\right] .
$$


where the real uncertainty parameters $\delta_{1}$ and $\delta_{2}$ vary from -1 to 1 , respectively.

The LPV model of the HiMAT vehicle is

$$
\begin{gathered}
\dot{x}=A x+B\left[\begin{array}{cc}
\bar{\tau}_{1} & 0 \\
0 & \bar{\tau}_{2}
\end{array}\right] u+B\left[\begin{array}{cc}
0.05 & 0 \\
0 & 0.05
\end{array}\right] w, \\
z=u, \quad y=C x \\
w=\Delta z, \quad \Delta=\left[\begin{array}{cc}
\delta_{1} & 0 \\
0 & \delta_{2}
\end{array}\right]
\end{gathered}
$$

The LPV model in equation (16) is a linear function of a parameter vector $\bar{\tau}$. However, the parameter vector $\tau$ can not be chosen as a scheduling parameter since we consider that the actuators are failed one at a time. Thus, $\bar{\tau}_{1}$ and $\bar{\tau}_{2}$ can not be zero simultaneously. To describe the failure cases, a synthetic scheduling parameter $\bar{\rho}$ is introduced as $0 \leq \bar{\rho} \leq 2$.

$$
\begin{array}{cccc}
0 \leq \bar{\rho}<1 & : & 0 \leq \bar{\tau}_{1}<1, & \bar{\tau}_{2}=1 \\
\bar{\rho}=1 & : & \bar{\tau}_{1}=1, & \bar{\tau}_{2}=1 \\
1<\bar{\rho} \leq 2 & : & \bar{\tau}_{1}=1, & 0 \leq \bar{\tau}_{2}<1
\end{array}
$$

Note that the LPV model of the HiMAT vehicle is not an affine function of a scheduling parameter $\bar{\rho}$.

\subsection{LPV Controller Design}

The control objective is to track a pitch angle command at actuator failure cases. A designed LPV controller should robustly stabilize the HiMAT vehicle over the failure parameter variations. The controller synthesis problem is formulated as a model matching problem in Figure 1. The ideal response

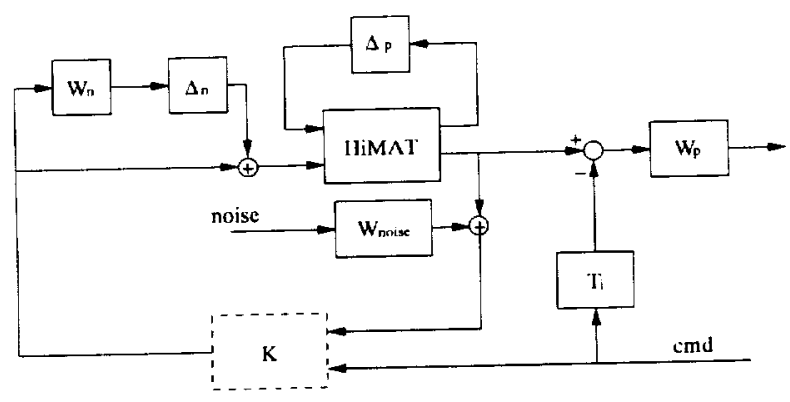

Figure 1: Interconnection structure for the model matching problem.

model $T_{i}$ of pitch angle is taken form the example in $\mu$-synthesis Toolbox. ${ }^{12}$ The performance weighting function $W_{p}$ and unmodeled dynamics $W_{n}$ are also taken from the example in $\mu$-synthesis Toolbox. ${ }^{12}$ The sensor noise is modeled as white noise with $0.6^{\circ}$ amplitude for angle of attack and pitch angle measurements. The weighting functions in Figure 1 are

$$
\begin{array}{ll}
T_{i} & =\frac{1}{\mathrm{~s} / 0.8+1}, \\
W_{p} & =40 \frac{\mathrm{s} / 50+1}{\mathrm{~s} / 0.05+1}, \\
W_{n} & =0.2 \frac{\mathrm{s} / 5+1}{\mathrm{~s} / 1000+1} I_{2 \times 2}, \\
W_{\text {noise }} & =0.01 I_{2 \times 2} .
\end{array}
$$

The control synthesis problem of the HiMAT vehicle is formulated to minimize the induced $-\mathcal{L}_{2}$ norm of the augmented LPV system with the weighting functions.

To solve the control synthesis LMI optimization problem in equation (4), basis functions for $X(\bar{\rho})$ and $Y(\bar{\rho})$ are required since $X$ and $Y$ are assumed as functions of $\bar{\rho}$.

$$
\begin{aligned}
& X(\bar{\rho})=\sum_{i} f_{i}(\bar{\rho}) X_{i}, \quad X_{i} \in \mathcal{R}^{n \times n}, \\
& Y(\bar{\rho})=\sum_{j} g_{j}(\bar{\rho}) Y_{j}, \quad Y_{j} \in \mathcal{R}^{n \times n} .
\end{aligned}
$$

where basis functions $f_{i}(\bar{\rho})$ and $g_{j}(\bar{\rho})$ are given before solving the LMI optimization in equation (4) over $X_{i}$ and $Y_{j}$. There is no analytic method to choose optimal basis functions for $X$ and $Y$ in general. In this paper, the basis function set is defined as $\{1,1 / \bar{\rho}, \bar{\rho}\}$ for $X$ and $Y$ to help the LMI optimization for total failure cases $\left(\bar{\tau}_{1}=0, \bar{\tau}_{2}=0\right)$, since the functions $f_{i}(\bar{\rho})$ and $g_{j}(\bar{\rho})$ are related with sensitivity of unknown matrices $X_{i}$ and $Y_{i}$, respectively. Note that it is not necessary to define that $g_{j}(\bar{\rho})$ is equal to $f_{i}(\bar{\rho})$.

Since $X$ and $Y$ are functions of $\bar{\rho}$, the parameter rate bound is required to solve the LMI optimization in equation (4). Recall that the scheduling parameter is an actuator failure parameter. Thus, for example, the scheduling parameter can suddenly vary from 1 (no failure case) to 2 (total canard failure case). In this paper, the parameter rate bound is assumed as $|\overline{\bar{\rho}}|<100$ to capture sudden variations of the scheduling parameter.

To make the LMI optimization computationally tractable, the LMI constraints are evaluated at the following grid points:

$$
\bar{\rho} \in\{\bar{\rho} \mid 0.01,0.1,0.2, \cdots, 1.9,2\} .
$$




\begin{tabular}{ccl}
\hline \hline Iteration & $\gamma$ & \multicolumn{1}{c}{$S$} \\
\hline 1 & 1.23 & $\operatorname{diag}([1,1,1,1])$ \\
2 & 0.71 & $\operatorname{diag}([0.497,0.168,1.186,1.277])$ \\
3 & 0.60 & $\operatorname{diag}([1.404,1.289,1.801,1.453])$ \\
4 & 0.54 & $\operatorname{diag}([1.580,2.193,2.100,1.187])$ \\
5 & 0.85 & $\operatorname{diag}([2.007,1.430,2.506,1.849])$ \\
\hline
\end{tabular}

Table 1: $\gamma$ values in the LMI optimization in equation (4).

Also, the same grid points are used to solve the LMI optimization in equation (5). Since the matrix $P$ is related with $X$ and $Y$, the basis function set for $P$ is chosen as $\{1,1 / \bar{\rho}, \bar{\rho}\}$.

In this paper, the scaling factor $S$ is assumed as constant over all scheduling parameter variations. The $\gamma$ values and the scaling factor $S$ for each iteration are written in Table 1 . The scaling factor $S$ is associated with the uncertainty block $\Delta$ which is

$$
\Delta=\operatorname{diag}\left(\left[\delta_{1}, \delta_{2}, \delta_{\text {ele }}, \delta_{\text {can }}\right]\right) .
$$

Recall that the real uncertainty parameters $\delta_{1}$ and $\delta_{2}$ are associated with elevon and canard actuator failure parameters, respectively. The multiplicative uncertainty parameters $\delta_{e l e}$ and $\delta_{c a n}$ are also associated with elevon and canard control channels in Figure 1 . The iteration process is stopped at the $5^{\text {th }}$ iteration since the $\gamma$ value at the iteration is greater than the previous iteration. Recall that the iteration process is not guaranteed to be converged. However, the performance index $\gamma$ in the LMI optimization of equation (4) is significantly reduced from 1.23 to 0.54 by using the scaling factor $S$. In the remainder of this paper, the LPV controller for the HiMAT vehicle denotes the designed LPV controller at the $4^{\text {th }}$ iteration.

\section{Simulations}

In this section, the designed LPV controller is applied to control the HiMAT vehicle for actuator failure cases. The HiMAT vehicle is simulated at a predefined failure scenario. One of the failure scenarios is defined as: the canards are failed from 1 to 10 seconds and the elevons are failed from 20 to 40 seconds. The actuator failure parameter for the failure scenario is set as:

$$
\rho= \begin{cases}0.01, & 1 \leq t<10 \mathrm{sec}, \\ 1.00, & 0 \leq t<1 \mathrm{sec}, \quad 10 \leq t \leq 20 \mathrm{sec} \\ 2.00, & 20<t \leq 40 \mathrm{sec} .\end{cases}
$$

For comparison, the LPV controller for the HiMAT vehicle is simulated with and without actuator failures. The simulation results are shown in Figure 2. The pitch angle commands are given as $10^{\circ}$ at $1 \mathrm{sec}$, $0^{\circ}$ at $10 \mathrm{sec}$, and $10^{\circ}$ at $20 \mathrm{sec}$, sequentially. In this
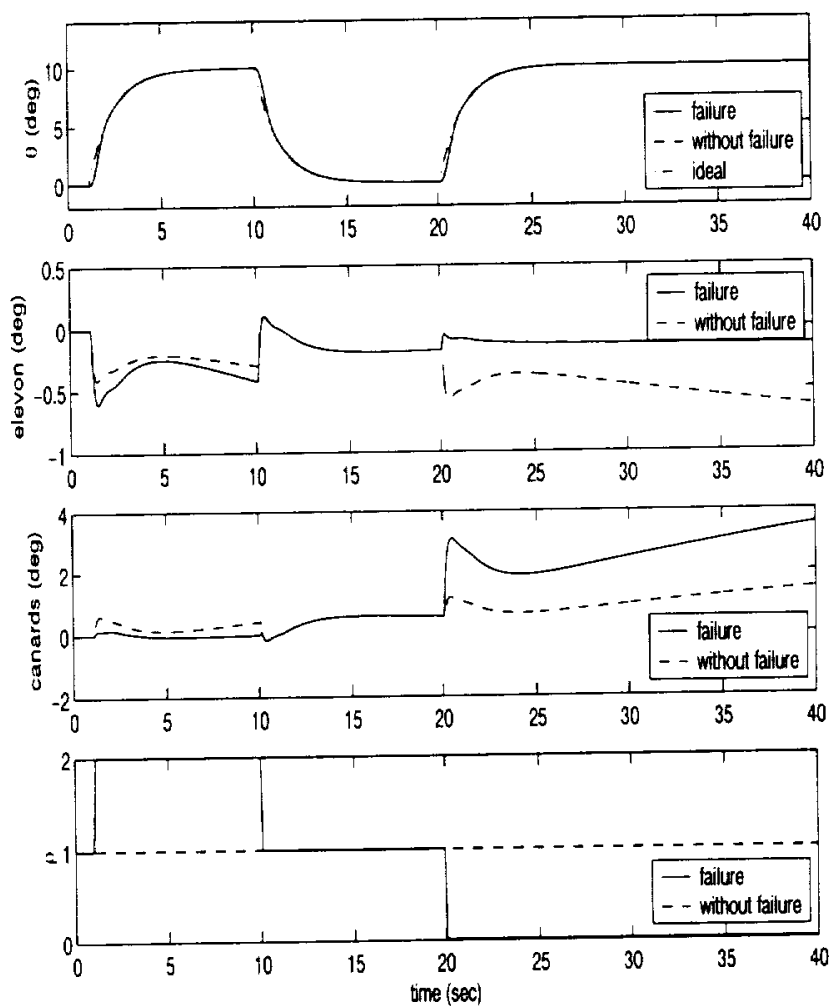

Figure 2: The LPV controller simulations with and without actuator failures.

simulation, we assumed that the failure parameter is measured as true value shown in the bottom plot in Figure 2. It is observed that the LPV controller achieves the desired performance level of tracking pitch commands at actuator failure cases. From the control signals for actuators in Figure 2, the LPV controller significantly uses the canard actuator to track the pitch commands when the elevon actuator is failed from $20 \mathrm{sec}$ to $40 \mathrm{sec}$. Also, the LPV controller keeps the elevon actuator signals close to zero 
at the elevon actuator failure case.

For the robustness of the LPV controller, the uncertainty parameters $\delta_{1}$ and $\delta_{2}$ are chosen as certain numbers such that $\Delta_{p}=\operatorname{diag}\left(\left[\delta_{1}, \delta_{2}\right]\right), \quad\left\|\Delta_{p}\right\| \leq 1$ and are folded into the LPV model of the HiMAT vehicle. The LPV controller is simulated with the perturbed system at the same failure scenario described in equation (21). Two examples of uncertainty parameters are shown in Figure 3 for space limitation. "pert1" and "pert2" in Figure 3 denote $\left[\begin{array}{ll}\delta_{1} & \delta_{2}\end{array}\right]=\left[\begin{array}{ll}1 & 1\end{array}\right]$ and $\left[\begin{array}{ll}\delta_{1} & \delta_{2}\end{array}\right]=\left[\begin{array}{ll}-1 & -1\end{array}\right]$, respectively. The simulation results show that the LPV controller can robustly stabilize the perturbed system and achieve the desired performance level of tracking the pitch angle commands.

For comparison, an $H_{\infty}$ controller as a linear time invariant controller for the HiMAT vehicle is designed at $\bar{\rho}=1$ (without failure cases) with the same weighting functions described in Section 4, using $\mu$ synthesis Toolbox. ${ }^{12}$ The $H_{\infty}$ controller is applied for the actuator failure scenario of equation (21). The bottom plots of Figure 3 show that the $H_{\infty}$ con-
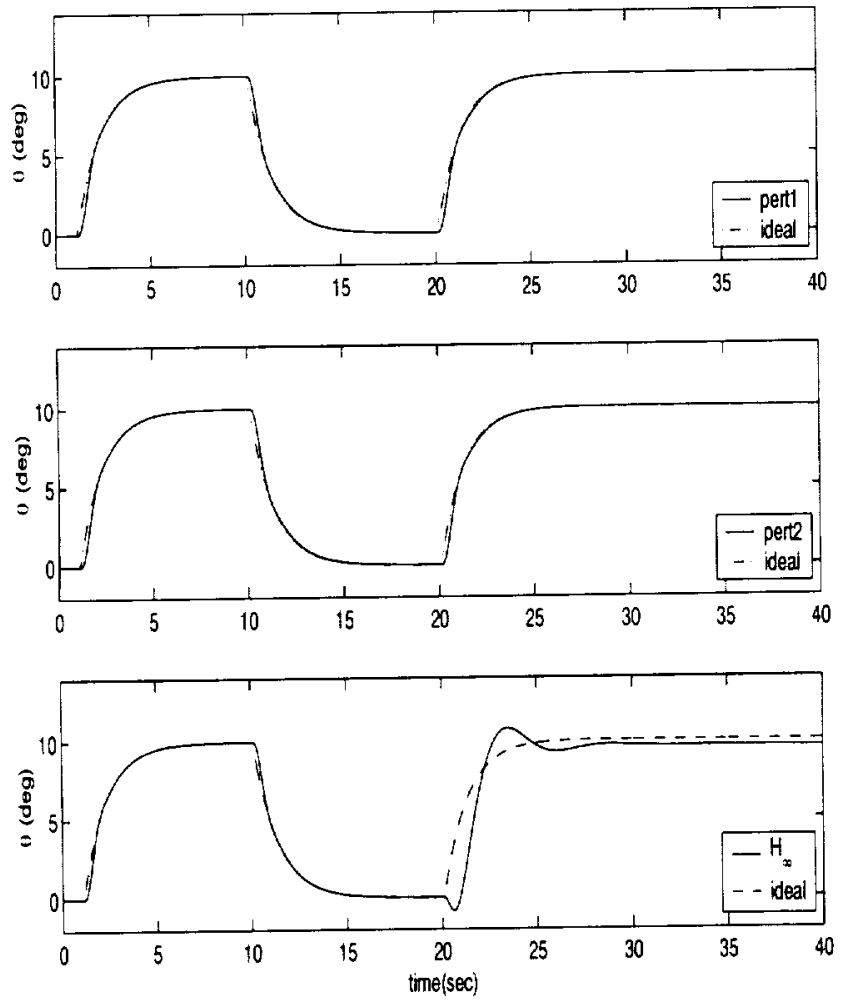

Figure 3: Time responses of pitch angle with LPV and $H_{\infty}$ controllers.

troller can achieve the desired performance level at a canard failure case $(1 \leq t<10 \mathrm{sec})$ even though the
$H_{\infty}$ controller is designed at $\bar{\rho}=1$. It shows that the $H_{\infty}$ controller inherently has some level of robustness. However, the $H_{\infty}$ controller can't achieve the desired performance level at the elevon failure case. Since the canards are less effective in controlling the pitch movement in comparison with elevons, ${ }^{16}$ the elevon failure can significantly affect on tracking the pitch commands.

The control signals of the LPV and $H_{\infty}$ controllers are shown in Figure 4 at the same failure scenario. Since the control signals of the LPV con-
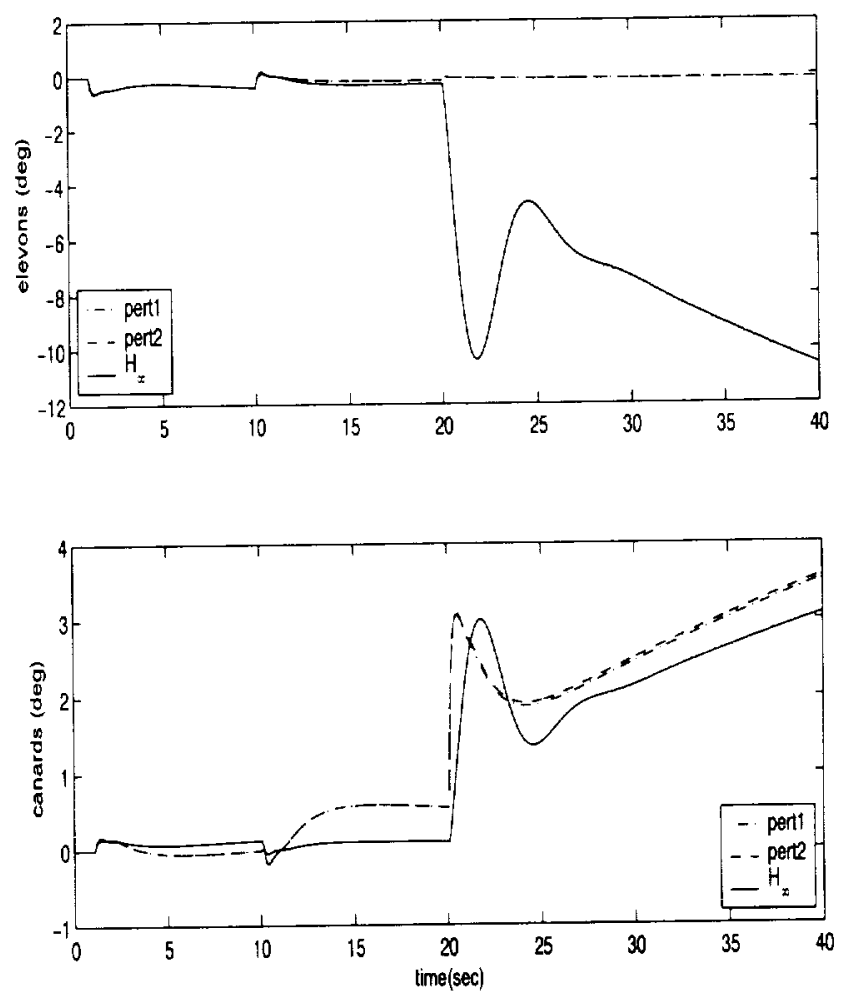

Figure 4: Control signals for each actuator with the LPV and $H_{\infty}$ controllers.

troller with/without perturbations of $\delta_{1}$ and $\delta_{2}$ are same, the "pert1" and "pert2" simulation results are plotted in Figure 4. It is observable from Figure 4 that the elevon signals of the $H_{\infty}$ controller are significantly increased at the elevon failure case. That is not appropriate to implement the $H_{\infty}$ controller into the real system. However, the LPV controller significantly reduces the elevon signals and increases the canard signals to compensate the elevon failure in Figure 4.

Now, the fault parameter is estimated with the on-line estimator integrated with the LPV controller shown in Figure 5. The on-line estimator in Figure 5 
has two parts: one is the two-stage discrete Kalman filter and the other is a simple logic in equation (19) to calculate the fault parameter from the bias estimates. To simulate the Kalman filter with the $\mathrm{Hi}$ MAT vehicle, several parameters should be defined. In this paper, sampling time is set as $0.01 \mathrm{sec}$ to

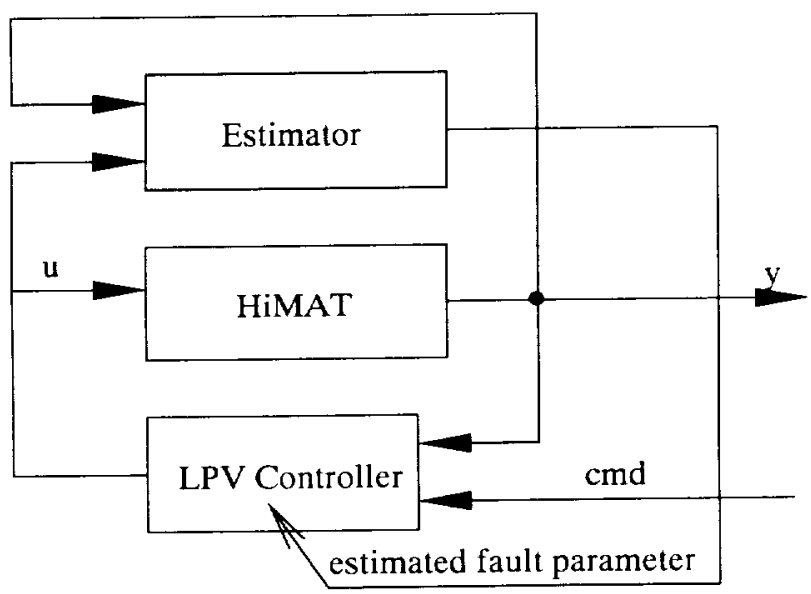

Figure 5: Simulation block diagram of a closed-loop system with an on-line estimator.

capture the open-loop dynamics of the vehicle. The covariance matrices $Q_{k}^{x}, Q_{k}^{\gamma}$ and $R_{k}^{\gamma}$ described in in Section 3 are set as constant matrices:

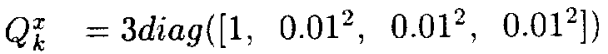

$$
\begin{aligned}
& Q_{k}^{\gamma}=3 \operatorname{diag}\left(\left[0.05^{2}, 0.05^{2}\right]\right) \\
& R_{k}^{\gamma}=3 \operatorname{diag}\left(\left[0.01^{2}, 0.01^{2}\right]\right)
\end{aligned}
$$

since the covariance matrices are related with convergence of the estimator and noise allowance considered in the control synthesis in Section 4. The initial values of estimated state $\hat{x}_{0 \mid 0}$ and biases $\hat{\gamma}_{0 \mid 0}$ are set as $\left[\begin{array}{llll}0 & 0 & 0 & 0\end{array}\right]^{T}$ and $\left[\begin{array}{ll}0 & 0\end{array}\right]^{T}$ with assumption of perfect estimation at $\mathrm{t}=0$. The initial covariance matrices $P_{0 \mid 0}^{\gamma}$ and $\tilde{P}_{0 \mid 0}^{x}$ are defined as $10 I_{2}$ and $10 I_{4}$. We choose the different values such as 0,100 for the initial covariance matrices for simulations. The bias estimates are not dependent on the initial values.

The most delicate parts of the bias estimation mechanism used in this paper are to define values of $\lambda_{0}, \alpha_{\min }$ and $\alpha_{\max }$ in equation (13) for covariance propagation. There is no a analytic way to define the values in general. Also, it is not known how the values affect on robustness of the closed-loop system. In this paper, we simulate the on-line bias estimator

\begin{tabular}{cccc}
\hline \hline Case & $\lambda_{0}$ & $\alpha_{\min }$ & $\alpha_{\max }$ \\
\hline 1 & 0.90 & 10 & 100 \\
2 & 0.95 & 10 & 100 \\
3 & 1.00 & 10 & 100 \\
4 & 1.00 & $10^{6}$ & $10^{7}$ \\
\hline
\end{tabular}

Table 2: The different sets of the varying forgetting factors

based on the different sets of the values in Table 2 .

The fault parameter on-line estimate results are
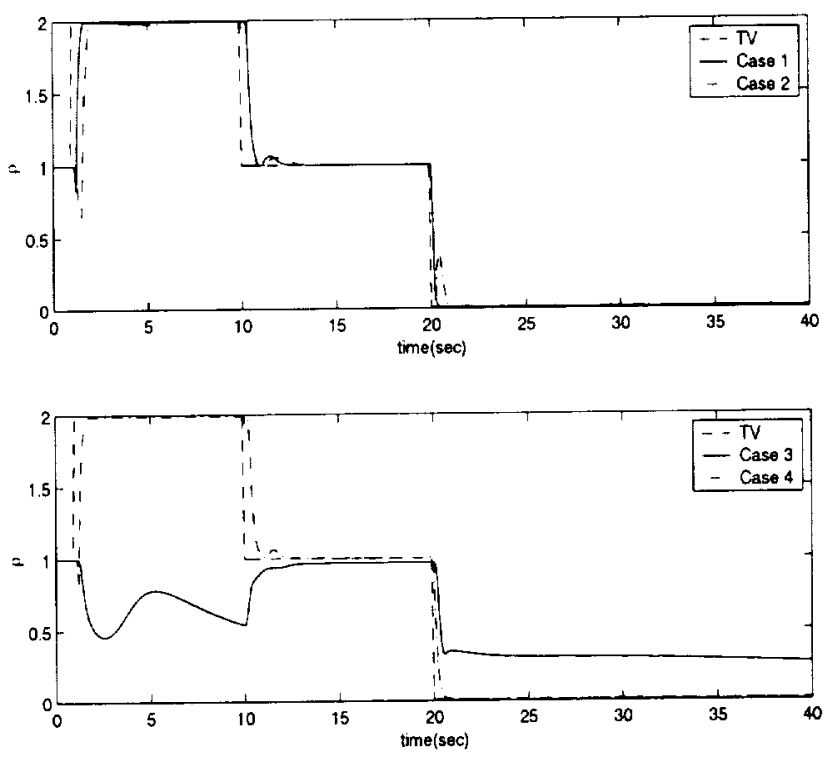

Figure 6: Fault parameter estimates according to the cases in Table 2.

shown in Figure 6. It is noticeable that bias estimates are dependent on the cases. From the top plots of Figure 6, it is noticed that the value of $\lambda_{0}$ affect on convergence rate of estimation. When $\lambda_{o}$ is set as 1 at Case 3, the bias estimate is not converged at canard failure situation. For Case 3, the fault parameter estimator returns false failure indication which is elevon failure instead of canard failure. Since the canards of the HiMAT vehicle have less controllability than the elevons do, it is much harder to detect the canard failure than to do the elevon failure. In Case 1,2,and 4, the false failure indication around $1 \mathrm{sec}$ is shown in Figure 6. For Case 4 , the covariance matrix $P^{\gamma}$ is immediately high value after one step integration since $\alpha_{\min }$ is 
defined as $10^{6}$. Case 4 is not realistic. However, it is easily noticed how $P^{\gamma}$ affects on the estimator.

Simulation results at Case 1 are shown in Figure 7. "TV" and "VFF" in Figure 7 denote that the LPV controller is evaluated at the true values (TV) of the failure parameter and at the estimated failure parameter with a variable forgetting factor (VFF), respectively. It is noticeable that the LPV controller evaluated at the estimated parameter can achieve the desired performance of tracking the pitch commands. The difference between estimated failure parameter and true failure parameter is very small at a steady state. However, there is a delay to estimate the failure parameter, which is not included in the LPV control synthesis process. The delay is help-
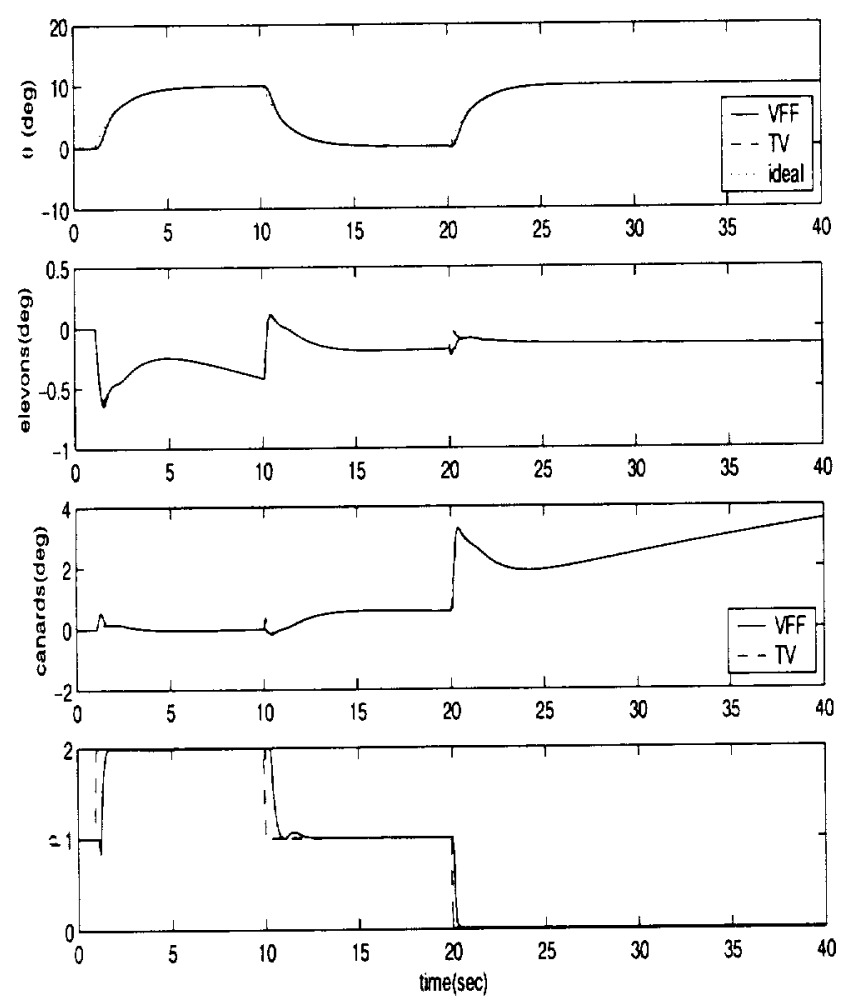

Figure 7: Simulations with the on-line estimator.

ful to make the scheduling parameter rate bounds which is one of the assumptions of the LPV control synthesis methodology. However, the large delay of fault parameter estimations is not appropriated for a fault tolerant control system. It is not studied here to include the delay term in the LPV control synthesis methodology. It is unknown to find out the optimal delay for reconfigurability of the LPV controller in the fault tolerant control system.

\section{Conclusion}

In this paper, the LPV controller is designed based on the estimated scheduling parameter. The system variations due to actuator failures are modeled as functions of estimated parameters and bounded parameter estimation errors represented by LFT form with the uncertainty block. An LPV controller synthesis problem with bounded parameter errors is formulated into the two LMI optimizations which are solved by the iteration method. The performance level of the closed-loop system with the designed LPV controller is reduced by the constant scaling factor on the uncertainty block.

The iteration approach of the LPV synthesis methodology is applied to control of the HiMAT vehicle at actuator failure cases. It is assumed that the actuators are failed one at a time. Actuator failure parameters of the HIMAT vehicle are estimated as biases using an augmented Kalman filter. The LPV controller evaluated at the estimated failure parameter is simulated with the HIMAT vehicle which varies as true values of the failure parameter. The simulation results show that the LPV controller achieves the desired performance level of tracking pitch angle commands for actuator failure cases.

\section{Acknowledgment}

This research was supported in part by National Aeronautics and Space Administration under NASA Contract No. NAS1-97046 and in part by by NASA under Cooperative Agreement NCC-1-02009 which is gratefully acknowledged by the authors.

\section{References}

[1] Blanke, M., Izadi-Zamanabadi, R., Bogh, S.A., and Lunau, C.P., "Fault-Tolerant Control System- A Holistic View," Control Engineering Control Practice, Vol. 5, No. 5, 1997, pp. 693 702 . 
[2] Chen, J., Patton, R.J., and Chen, Z, "An LMI approach to Fault-Tolerant Control of Uncertain System," in Proceeding of the 1999 IEEE/ISIC/CIRA/ISAS Joint Conference, 1998, pp. 175-180.

[3] Ganguli, S., Marcos, A., and Balas, G., "Reconfigurable LPV Control Design for Boeing 747100/200 Longitudinal Axis," in Proceedings of American Control Conference, 2002, pp. 3612 3617 .

[4] Apkarian, P. and Adams, R., "Advanced GainScheduling Techniques for Uncertain Systems," IEEE Transactions on Control Systems Technology, Vol. 6, No. 1, 1998, pp. 21-32.

[5] Wu, F., Control of Linear Parameter Varying Systems. PhD thesis, Department of Mechanical Engineering, University of California, Berkeley, 1995.

[6] Wu, F., Packard, A., and Balas, G., "LPV Control Design for Pitch-Axis Missile Autopilots," in Proceedings of the 34th IEEE Conference on Decision and Control, (New Orleans, LA), 1995, pp. 188-193.

[7] Shamma, J., "Gain-Scheduled Missile Autopilot Design Using Linear Parameter Varying Transformations," Journal of Guidance, Control, and Dynamics, Vol. 16, No. 2, 1993, pp. 256-261.

[8] Balas, G., Fialho, I., Packard, A., Renfrow, J., and Mullaney, C., "On the Design of LPV Controllers for the F-14 Aircraft Lateral-Directional Axis During Powered Approach," in Proceedings of the American Control Conference, (A1buquerque, NM), 1997, pp. 123-127.

[9] Balas, G., Ryan, J., Shin, J-Y., and Garrard, W., "A New Technique for Design of Controllers for Turbofan Engines," in AIAA 34th Join Propulsion Conference, (Cleveland, $\mathrm{OH}$ ), 1998, pp. 1-6. AIAA-98-3751.

[10] Shin, J.-Y., Worst-Case Analysis and Linear Parameter-Varying Gain-Scheduled Control of Aerospace Systems. PhD thesis, Department of Aerospace Engineering and Mechanics, University of Minnesota, 2000.

[11] Wu, N. Eva, Zhang, Y., and Zhou, K., "Detection, Estimation, and Accommodation of Loss of Control Effectiveness," International Journal of Adaptive Control and Signal Processing, Vol. 14, 2000, pp. 775-795.
[12] Balas, G., Doyle, J., Glover, K., and Packard, A., $\mu$ Analysis and Synthesis Toolbox. Natick, MA: The Mathworks, Inc., 1995.

[13] Becker, G., Quadratic Stability and Performance of Linear Parameter Dependent Systems. PhD thesis, Department of Mechanical Engineering, Cniversity of California, Berkeley, 1993.

[14] Keller, J. K. and Darouach, M., "Optimal twostage Kalman filter in the presence of random bias," Automatica, Vol. 33, 1997, pp. 17451748 .

[15] Parkum, J. E., Poulsen, N. K., and Holst, J., "Recursive forgetting algorithms," International Journal of Control, Vol. 55, 1992, pp. 109-128.

[16] Wu, N. Eva, Zhou, K., and Saloman, G., "Control Reconfigurability of Linear Time-Invariant Systems," Automatica, Vol. 36, 2000, pp. 17671771. 


\title{
LINEAR PARAMETER VARYING CONTROL SYNTHESIS FOR ACTUATOR FAILURE, BASED ON ESTIMATED PARAMETER *
}

\author{
Jong-Yeob Shin, ${ }^{\dagger}$ N. Eva Wu, ${ }^{\ddagger}$ and Christine Belcastro ${ }^{\S}$
}

\begin{abstract}
A robust linear parameter varying (LPV) control synthesis is carried out for an HiMAT vehicle subject to loss of control effectiveness. The scheduling parameter is selected to be a function of the estimates of the control effectiveness factors. The estimates are provided on-line by a two-stage Kalman estimator. The inherent conservatism of the LPV design is reduced through the use of a scaling factor on the uncertainty block that represents the estimation errors of the effectiveness factors. Simulations of the controlled system with the on-line estimator show that a superior fault-tolerance can be achieved.
\end{abstract}

\section{Introduction}

One of the most effective methods for achieving single aircraft accident prevention ${ }^{1}$ is to design flight control laws that are fault-tolerant. An active faulttolerant control (FTC) system requires its control law to react to actuator/sensor faults through reconfiguration. It is important that the design of a fault detection and isolation (FDI) mechanism in an FTC system be properly integrated with the design of a fault tolerant control mechanism. ${ }^{2}$

Fault-tolerant control designs for aerospace vehicles based on linear matrix inequality (LMI) optimization solutions can be found in several reports. For example, in Ref.[3,4], fault tolerant controllers

\footnotetext{
${ }^{*}$ Copyright (C) 2002 by J-Y. Shin, N. E. Wu, and C. Bel castro. Published by the American Insitute of Aeronautics and Astronautics, Inc. with permission.

† taff Scientist, ICASE, NASA Langley Research Center, Hampton, VA 23681. AIAA Member

¥ Professor, Department of Electrical Engineering, Binghamton State University of New York, Binghamton, New York, 13902.

$\$$ Researcher, Guidance Control Brench, NASA Langley Research Center, Hampton, VA 23681.
}

are designed based on information provided by separate FDI modules that are assumed to have identified faults, while faults are modeled as functions of model parameters. In particular, when faults are modeled as a set of varying parameters in a linear parameter varying (LPV) system, a known LPV control synthesis method ${ }^{5,6}$ can be attempted. The synthesis is formulated into an LMI optimization problem based on the LPV system whose statespace matrices are functions of a scheduling parameter vector. A scheduling parameter vector must be measurable in real time and its rate of change is required to be bounded..$^{5,6}$ It is possible that an LPV controller can robustly stabilize a close-loop system and achieve a desired performance level over the entire parameter space. The LPV synthesis methodology has been successfully applied to synthesize controllers for the pitch-axis missile autopilots, ${ }^{7,8}$ the $\mathrm{F}$ 14 aircraft lateral-directional axis using powered approach,${ }^{9}$ turbofan engines ${ }^{10}$ and the F-16 aircraft. ${ }^{11}$

In this paper, fault parameters are considered as scheduling parameters in LPV control synthesis, whose rate of change is defined as a sufficiently large number but not infinite. In practical, the bounded large number of scheduling parameter rate of change can allow abrupt fault occurrence in a reconfigurable LPV controller in Ref. $\left[{ }^{4}\right]$. Since the fault parameters cannot be directly measured via sensors in real time, they are estimated on-line instead using a two-stage adaptive Kalman filtering scheme. ${ }^{12}$ To deal with the uncertainty due to estimation errors which always exist in practice, in this paper, modeling of fault parameter estimation error is also included as the part of the problem formulation in the form of an uncertainty block, and this estimation error enters in the construction of an LPV controller. Thus, the LPV controller in an FTC system can allow to be easily integrated with an FDI system-the on-line parameter estimator. The LPV control synthesis problem can be formulated into a robust LPV control synthesis. ${ }^{5}$ In this paper, the robust LPV control

1

American Institute of Aeronautics and Astronautics 